\title{
On the Green Function of the Landau Operator and its Properties Related to Point Interactions
}

\author{
V. A. Geyler and V. V. Demidov
}

\begin{abstract}
The Green function $G$ of the Schrödinger operator with a magnetic field (i.e. the Landau operator) $H$ is studied. Two representations of $G$ are used, namely in form of an integral and of a series. The space-variable asymptotics as well as the energetic ones are obtained. The analytical and asymptotical properties of $G$ we obtain are related to point perturbations of $H$.
\end{abstract}

Keywords: Schrödinger operator, magnetic fields, Green functions, zero-range potentials AMS subject classification: $35 \mathrm{~J} 10$

\section{Introduction}

By a Landau operator, we mean a three-dimensional Schrödinger operator $H$ with a uniform magnetic field $B$ (see [5: Subsection 1.3]). After the discovery of quantum Hall effect by $K$. von Klitzing [19], periodic and stochastic perturbations of the Landau operator become the subject of intensive studies both in mathematics and in theoretical physics (see, e.g., $[3,5-7,23,25]$ and the references therein). A number of results explaining some properties of quantum Hall systems has been obtained by means of point perturbations of the Landau operators (see $[2,8,11,12,14,16]$ ) etc. The simplest way to strict mathematical justification of such results is based on the use of the Krein resolvent formula (see $[1 ; 21,23]$ ). The possibility of this use takes place only if the Green function $G$ of the unperturbed operator possesses some analytical and asymptotical properties. Our first goal in the present paper is to show that the Green function of the Landau operator has properties required for existence of the Green function of the operator $H$ perturbed by a zero-range potential supported on an infinite discrete set [13] (see Theorem 3 and equation (5.5)).

The advantage of zero-range potentials is the possibility to find the spectral parameters of perturbed operators in an explicit form. For this purpose explicit formulae for the Green function $G$ are required. Our second goal is to derive such formulae (see Theorems 1 and 2). In this connection we give direct proofs of the main results using the formulae for $G$, although certain of the proofs can be obtained by the use of general properties of pseudo-differential operators [126]. Some results of the present paper have been announced without proofs in $[10,13]$.

V. A. Geyler: Ogarev Univ. Mordovia, Dept. Math., Saransk 430000, Russia

V. V. Demidov: Ogarev Univ. Mordovia, Dept. Math., Saransk 430000, Russia

ISSN 0232-2064 / \$2.50 C Heldermann Verlag Berlin 


\section{Preliminary}

The self-adjoint operator $H$ we consider is the closure of the following symmetric operator defined on the space $C_{0}^{\infty}\left(\mathbb{R}^{3}\right)$ :

$$
H_{0}=\left(-i \frac{\partial}{\partial x_{1}}+\pi \xi x_{2}\right)^{2}+\left(i \frac{\partial}{\partial x_{2}}+\pi \xi x_{1}\right)^{2}-\frac{\partial^{2}}{\partial x_{3}^{2}}
$$

(see, e.g., [5: Subsection 1.3]). Here $\xi>0$ is the number of magnetic flux quanta through a unit area of the $\left(x_{1}, x_{2}\right)$-plane and the magnetic field $B$ is derected along the $x_{3}$-axis. It is well known that $H$ has absolutely continuous spectrum $\sigma(H)$ and that $\sigma(H)=\left[\frac{\omega}{2},+\infty\right)$ where $\omega=4 \pi \xi$. Using the Kato inequality (see [18: Theorem $2]$ ), it is easy to show that the domain of $H$ is a subset of $C\left(\mathbb{R}^{3}\right)$. Hence the resolvent $R(\zeta)=(H-\zeta)^{-1}$ of $H$ is a Carleman operator, i.e. $R(\zeta)$ has a measurable integral kernel $G(x, y ; \zeta)$ obeying

$$
\int_{\mathbb{R}^{3}}|G(x, y ; \zeta)|^{2} d y<\infty
$$

for almost all $x \in \mathbb{R}^{3}$ and for all $\zeta \in \rho(H)=\mathbb{C} \backslash \sigma(H)$ (see [20: Chapter III, Theorem $4.2])$.

At the beginning we recall some relevant properties of the two-dimensional Landau operator $H^{(2)}$ which is by definition the closure of the symmetric operator $H_{0}^{(2)}$ defined on the space $C_{0}^{\infty}\left(\mathbb{R}^{2}\right)$ by the relation

$$
H_{0}^{(2)}=\left(-i \frac{\partial}{\partial x_{1}}+\pi \xi x_{2}\right)^{2}+\left(i \frac{\partial}{\partial x_{2}}+\pi \xi x_{1}\right)^{2}
$$

(sec, e.g., [9: Subsection 1.1]). The spectrum of $H^{(2)}$ is a pure point one and consists of infinitely degenerate eigenvalues (the Landau levels) $\varepsilon_{l}=\omega\left(l+\frac{1}{2}\right) \cdot(l \geq 0)$. The Green function $G^{(2)}(x, y ; \zeta)$ of the operator $H^{(2)}$ has the explicit form

$$
\begin{aligned}
G^{(2)}(x, y ; \zeta)= & \frac{1}{4 \pi} \Gamma\left(\frac{1}{2}-\frac{\zeta}{\omega}\right) \exp \left[-i \pi \xi x \wedge y-\pi \xi \frac{(x-y)^{2}}{2}\right] \\
& \times \Psi\left(\frac{1}{2}-\frac{\zeta}{\omega}, 1 ; \pi \xi(x-y)^{2}\right)
\end{aligned}
$$

where $\Gamma$ is the Euler $\Gamma$-function, $\Psi$ is the confluent hypergeometric function (see [4: Vol. 1 , Subsection 6.5]) and the symbol $\wedge$ denotes the standard symplectic multiplication in $\mathbb{R}^{2}$, i.e. $x \wedge y=x_{1} y_{2}-x_{2} y_{1}$ for $x, y \in \mathbb{R}^{2}$ with $x=\left(x_{1}, x_{2}\right)$ and $y=\left(y_{1}, y_{2}\right)$. In connection with (2) we shall need the formula (see [24: 'Vol.1/Formula 6.5(2)])

$$
\Gamma(a) \Psi(a, 1 ; x)=\int_{0}^{\infty} e^{-x t}\left(\frac{t}{1+t}\right)^{a} \frac{d t}{t} \quad(x>0, \operatorname{Re} a>0)
$$


The orthogonal projection $P_{l}$ onto the eigenspace of $H^{(2)}$ corresponding to the eigenvalue $\varepsilon_{l}$ is an integral operator with kernel

$$
P_{l}(x, y)=\xi \exp \left[-i \pi \xi x \wedge y-\pi \xi \frac{(x-y)^{2}}{2}\right] L_{l}\left(\pi \xi(x-y)^{2}\right)
$$

where $L_{l}$ is the $l$-th Laguerre polynomial (see [4: Vol. 2, Subsection 10.12]).

Throughout the paper we consider the continuous branch of the square root $\sqrt{\zeta}=$ $\zeta^{1 / 2}$ on the complex plane $\mathbb{C}$ with cut along the negative real semi-axis $x \leq 0$ defined by the condition $\operatorname{Re} \sqrt{\zeta}>0$. We shall use the notation $x_{\perp}$ for the orthogonal projection of vector $x$ on the $\left(x_{1}, x_{2}\right)$-plane. The symbol $\Phi$ denotes the function

$$
\Phi(x, y)=\exp \left[-i \pi \xi\left(x_{\perp} \wedge y_{\perp}\right)-\pi \xi \frac{\left(x_{\perp}-y_{\perp}\right)^{2}}{2}\right]
$$

and the symbol $\mathbb{R}_{\Delta}^{6}$ the domain

$$
\mathbb{R}_{\Delta}^{6}=\left\{(x, y) \in \mathbb{R}^{3} \times \mathbb{R}^{3}: x \neq y\right\}
$$

\section{The series representation of $G(x, y ; \zeta)$}

The following theorem is the main result of this section.

Theorem 1. The following assertions are valid:

(i) For every $\zeta \in \rho(H)$,

$$
\begin{aligned}
G(x, y ; \zeta)= & \sqrt{\frac{\xi}{16 \pi}} \Phi(x, y) \\
& \times \sum_{l=0}^{\infty} \frac{\exp \left[-\left(\omega\left(l+\frac{1}{2}\right)-\zeta\right)^{\frac{1}{2}}\left|x_{3}-y_{3}\right|\right]}{\left(l+\frac{1}{2}-\frac{\zeta}{\omega}\right)^{\frac{1}{2}}} L_{l}\left(\pi \xi\left(x_{\perp}-y_{\perp}\right)^{2}\right)
\end{aligned}
$$

where the series converges locally uniformly in the domain $\mathbb{R}_{\Delta}^{6} \times \rho(H)$. Hence the function $G$ is continuous in this domain, and for every fixed $(x, y) \in \mathbb{R}_{\Delta}^{6}$ the function $G(x, y ; \cdot)$ is holomorphic in $\rho(H)$.

(ii) If $\varepsilon_{l} \neq \zeta_{0} \in \sigma(H)(l \geq 0)$, then for every $(x, y) \in \mathbb{R}_{\Delta}^{6}$ there exist the limits

$$
\lim _{\substack{\zeta \rightarrow \zeta_{0} \\ \operatorname{Re} \zeta>0}} G(x, y ; \zeta)=G^{+}\left(x, y ; \zeta_{0}\right) \quad \text { and } \quad \lim _{\substack{\zeta \rightarrow \zeta 0 \\ \operatorname{Ro} \zeta<0}} G(x, y ; \zeta)=G^{-}\left(x, y ; \zeta_{0}\right)
$$

which are continuous functions in $(x, y) \in \mathbb{R}_{\Delta}^{6}$.

(iii) For every $\zeta_{0} \in \rho(H)$ the function $\frac{\partial G\left(\cdot, \cdot \zeta_{0}\right)}{\partial \zeta}$ is continuable to a continuous function on the whole space $\mathbb{R}^{3} \times \mathbb{R}^{3}$.

The proof of the theorem is based on the lemma below. 
Lemma 1. Consider the series

$$
S(x ; \zeta)=\sum_{n=0}^{\infty} \frac{\exp \left(-\sqrt{n-\zeta}\left|x_{3}\right|\right)}{\sqrt{n-\zeta}} L_{n}\left(\rho^{2}\right) \quad\left(\rho^{2}=x_{1}^{2}+x_{2}^{2}\right)
$$

and the formal termwise derivative of $S(x ; \zeta)$ with respect to $\zeta$

$$
S^{\prime}(x ; \zeta)=\frac{1}{2} \sum_{n=0}^{\infty}\left[\frac{\exp \left(-\sqrt{n-\zeta}\left|x_{3}\right|\right)}{(n-\zeta)^{\frac{3}{2}}}+\frac{\left|x_{3}\right| \exp \left(-\sqrt{n-\zeta}\left|x_{3}\right|\right)}{n-\zeta}\right] L_{n}\left(\rho^{2}\right) .
$$

The following assertions are valid: $\mathbb{R}_{+}$).

(i) The series $S$ and $S^{\prime}$ converge locally uniformly in the domain $\left(\mathbb{R}^{3} \backslash\{0\}\right) \times(\mathbb{C} \backslash$

(ii) If $\zeta_{0} \in \mathbb{R}_{+} \backslash \mathbb{N}$, then there exist the limits

$$
\lim _{\substack{\zeta \rightarrow c_{0} \\ \operatorname{Re} \zeta>0}} S(x ; \zeta)=S^{+}\left(x ; \zeta_{0}\right) \quad \text { and } \quad \lim _{\substack{\zeta \rightarrow \zeta_{0} \\ \operatorname{Re}<<0}} S(x ; \zeta)=S^{-}\left(x ; \zeta_{0}\right)
$$

(iii) For every $\zeta \in \mathbb{C} \backslash \mathbb{R}_{+}$there exists the limit $\lim _{x \rightarrow 0} S^{\prime}(x ; \zeta)$.

Proof. Assertion (i): Let $0 \neq \tilde{x} \in \mathbb{R}^{3}$. Since

$$
\left|L_{n}(x)\right| \leq e^{x / 2} \quad(n \in \mathbb{N}, x \geq 0)
$$

(see [4: Vol.2/Formula 10.18(3)]), the assertion is evident if $\tilde{x}_{3} \neq 0$. Let $\tilde{x}_{3}=0$, and hence $\tilde{\rho}>0$. Denote $\delta_{1}=\frac{\tilde{\rho}}{2}$ and $\delta_{2}=2 \tilde{\rho}$. Then

$$
L_{n}\left(\rho^{2}\right)=\pi^{-\frac{1}{2}} e^{\frac{\rho^{2}}{2}} \rho^{-\frac{1}{2}} n^{-\frac{1}{4}} \cos \left(2 \sqrt{n} \rho-\frac{\pi}{4}\right)+O\left(n^{-\frac{3}{4}}\right)
$$

uniformly as $\rho$ runs through $\left[\delta_{1}, \delta_{2}\right]$ (see [23: Vol.2/ Formula 10.15(1)]). Equation (9) shows immediately that the series (7) converges uniformly in the region $\left|x_{3}\right| \leq 1$, $\rho \in\left[\delta_{1}, \delta_{2}\right]$ and $\zeta \in K$ where $K$ is an arbitrary compact of $\mathbb{C} \backslash \mathbb{R}_{+}$. Since the domain $\mathbb{C} \backslash \mathbb{R}_{+}$is connected, it remains to show that for some fixed $0<-\zeta_{0} \in \mathbb{R}$ the series $S\left(x ; \zeta_{0}\right)$ converges uniformly with respect to $x$ in the region $\left|x_{3}\right| \leq 1$ and $\rho \in\left[\delta_{1}, \delta_{2}\right]$. For this purpose we write the series $S\left(x ; \zeta_{0}\right)$ in the form

$$
S(x)=\sum_{n=0}^{\infty} a_{n}\left(x_{3}\right) b_{n}(\rho)
$$

where

$$
a_{n}\left(x_{3}\right)=\frac{n^{\frac{3}{8}} \exp \left(-\sqrt{n-\zeta_{0}}\left|x_{3}\right|\right)}{\sqrt{n-\zeta_{0}}} \quad \text { and } \quad b_{n}(\rho)=\frac{L_{n}(\rho)}{n^{\frac{3}{8}}} .
$$

We shall prove uniform convergence of the series (10) in the region $\left|x_{3}\right| \leq 1$ and $\rho \epsilon$ $\left[\delta_{1}, \delta_{2}\right]$ via the Abel-Dirichlet criterion. First we show that the sequence $\left\{a_{n}\left(x_{3}\right)\right\}_{n \geq 0}$ 
decreases for sufficiently large indices $n$ and tends to zero uniformly with respect to $x_{3}$. Denote

$$
f(\lambda)=\frac{\lambda^{\frac{3}{8}} \exp \left(-\sqrt{\lambda-\zeta_{0}}\left|x_{3}\right|\right)}{\sqrt{\lambda-\zeta_{0}}} \quad\left(\lambda>\zeta_{0}\right) .
$$

It is easy to show that $f^{\prime}(\lambda)<0$ if $\lambda$ is sufficiently large. Therefore there is an integer $n_{0}$ such that the sequence $\left\{a_{n}\left(x_{3}\right)\right\}_{n \geq n_{0}}$ decreases. As $\lim _{n \rightarrow \infty} a_{n}\left(x_{3}\right)=0$ for all $x_{3} \in[-1,1]$, the sequence $\left\{a_{n}\left(x_{3}\right)\right\}_{n \geq 0}$ converges uniformly with respect to $x_{3}$ by the Dini theorem.

Let us prove now uniform boundedness of the partial sums of the series $\sum b_{n}(\rho)$. By virtue of equation (9) it suffices to prove uniform boundedness of the partial sums of the series

$$
\sum_{n \geq 0} \frac{\cos \left(2 \sqrt{n} \rho-\frac{\pi}{4}\right)}{n^{\frac{5}{8}}}
$$

in $\left[\delta_{1}, \delta_{2}\right]$. By the Euler-Maclaurin formula [22: Subsection 8.2] we have

$$
\begin{aligned}
\sum_{n=n_{0}}^{m} \frac{\cos \left(2 \sqrt{n} \rho-\frac{\pi}{4}\right)}{n^{\frac{5}{8}}}= & \int_{n_{0}}^{m} \frac{\cos \left(2 \sqrt{x} \rho-\frac{\pi}{4}\right)}{x^{\frac{5}{8}}} d x \\
& +\frac{1}{2}\left[\frac{\cos \left(2 \sqrt{n_{0}} \rho-\frac{\pi}{4}\right)}{n_{0}^{\frac{5}{8}}}+\frac{\cos \left(2 \sqrt{m} \rho-\frac{\pi}{4}\right)}{m^{\frac{5}{8}}}\right]+R_{1}(m)
\end{aligned}
$$

where the remainder $R_{1}(m)$ admits the estimate

$$
\begin{aligned}
\left|R_{1}(m)\right| & \leq \frac{1}{8} \int_{n_{0}}^{m}\left|\left(\frac{\cos \left(2 \sqrt{x} \rho-\frac{\pi}{4}\right)}{x^{\frac{3}{8}}}\right)^{\prime \prime}\right| d x \\
& \leq \frac{\delta_{2}^{2}}{32} \int_{n_{0}}^{+\infty} \frac{d x}{x^{\frac{13}{8}}}+\frac{7 \delta_{2}}{64} \int_{n_{0}}^{+\infty} \frac{d x}{x^{\frac{17}{8}}}+\frac{65}{512} \int_{n_{0}}^{+\infty} \frac{d x}{x^{\frac{21}{8}}} .
\end{aligned}
$$

To estimate the integral

$$
I=\int_{n_{0}}^{m} x^{-\frac{5}{8}} \cos \left(2 \sqrt{x} \rho-\frac{\pi}{4}\right) d x
$$

changing $\sqrt{x}$ by the variable $t$ we obtain

$$
I=2 \int_{\sqrt{n_{0}}}^{\sqrt{m}} t^{-\frac{1}{4}} \cos \left(2 t \rho-\frac{\pi}{4}\right) d t .
$$

Since $t^{-\frac{1}{4}}$ decreases monotonically to zero, the expressions

$$
\left|\int_{\sqrt{n_{0}}}^{s} t^{-\frac{1}{4}} \cos \left(2 t \rho-\frac{\pi}{4}\right) d t\right|
$$


are bounded uniformly with respect to $s$ and $\rho$ in the region $s>\sqrt{n_{0}}$ and $\rho \in\left[\delta_{1}, \delta_{2}\right]$ (by the Abel-Dirichlet criterion for uniform convergence). Thus assertion (i) of the lemma is proved.

Assertion (ii): It is sufficient to note that repeating the previous arguments we can prove the following statement:

Let $\zeta_{0} \in \mathbb{R}_{+} \backslash \mathbb{N}$ and $x_{0} \in \mathbb{R}^{3} \backslash\{0\}$. Then there exist a neighbourhood $U$ of $x_{0}, a$ neighbourhood $V_{+}$of $\zeta_{0}$ in the closed upper half-plane and a neighbourhood $V_{-}$of $\zeta_{0}$ in the closed lower half-plane such that the series $S$ converges uniformly in the set $U \times V_{+}$ or in the set $U \times V_{-}$if an appropriate choice of a continuous branch of the square root in the corresponding neighbourhood of $\zeta_{0}$ is performed.

Assertion (iii): It suffices to verify that

$$
\lim _{0 \neq x_{3} \rightarrow 0} \sum_{n \geq 0} \frac{\left|x_{3}\right| \exp \left(-\sqrt{n-\zeta}\left|x_{3}\right|\right)}{|n-\zeta|}=0
$$

locally uniformly with respect to $\zeta \in \mathbb{C} \backslash \mathbb{R}_{+}$(remember that $\left|L_{n}\left(\rho^{2}\right)\right| \leq e^{\frac{e^{2}}{2}}$ ). Let us consider the expression

$$
\frac{\exp \left(-\sqrt{n-\zeta}\left|x_{3}\right|\right)}{|n-\zeta|} \frac{n}{\exp \left(-\sqrt{n}\left|x_{3}\right|\right)}=\frac{n}{|n-\zeta|} \exp \left[(-\sqrt{n-\zeta}+\sqrt{n})\left|x_{3}\right|\right]
$$

It is clear that this expression tends to one locally uniformly with respect to $\zeta \in \mathbb{C} \backslash \mathbb{R}_{+}$ and uniformly with respect to $x_{3},\left|x_{3}\right| \leq 1$. Let now $\zeta_{0} \notin \mathbb{R}_{+}$be given. Then there exists a neighbourhood $V$ of $\zeta_{0}$ such that for all $\zeta \in V$ we have

$$
\left|\frac{\exp \left(-\sqrt{n-\zeta}\left|x_{3}\right|\right)}{n-\zeta}\right| \leq C \frac{\exp \left(-\sqrt{n}\left|x_{3}\right|\right)}{n}
$$

for some $C>0$. As $\frac{\exp \left(-\sqrt{n}\left|x_{3}\right|\right)}{n}$ decreases monotonically by $n$, it is sufficient to prove that

$$
\lim _{0 \neq x_{3} \rightarrow 0}\left|x_{3}\right| \int_{1}^{+\infty} \frac{\exp \left(-\sqrt{t}\left|x_{3}\right|\right)}{t} d t=0 \text {. }
$$

The change of variable $\frac{t}{\left|x_{3}\right|^{2}}=\tau$ yields

$$
\left|x_{3}\right| \int_{1}^{+\infty} \frac{\exp \left(-\sqrt{t}\left|x_{3}\right|\right)}{t} d t \leq-2\left|x_{3}\right| \ln \left|x_{3}\right|+\left|x_{3}\right| \int_{1}^{+\infty} \frac{\exp (-\sqrt{\tau})}{\tau} d \tau
$$

for $\left|x_{3}\right|<1$. Obviously, (12) implies (11), and the lemma is proved 
Proof of Theorem 1. It is evident that the operator $H$ is the sum of two operators

$$
H=H^{(2)} \otimes I_{x_{3}}+I_{x_{1}, x_{2}} \otimes\left(-\Delta_{x_{3}}\right)
$$

where $\Delta_{x_{3}}=\frac{d^{2}}{d x_{3}^{2}}$ and $I$ is the identity operator in the corresponding subspace. By virtue of (13), we obtain the resolvent $R(\zeta)$ as sum of the series

$$
R(\zeta)=\sum_{l=0}^{\infty} P_{l} \otimes\left(-\Delta_{x_{3}}+\varepsilon_{l}-\zeta\right)^{-1}
$$

which converges in the strong operator topology. It is well-known that the second factor in the sum (14) has the integral kernel

$$
\left(-\Delta_{x_{3}}-\zeta\right)^{-1}(x, y)=\frac{1}{2 \sqrt{-\zeta}} \exp (-\sqrt{-\zeta}|x-y|) .
$$

From (14), (4), (5) and (15) we obtain equation (6) (in the sense of strong operator topology). Therefore the statements of the theorem follow from the corresponding statements of Lemma 1

Remarks. (1) Strictly speeking, the Green function $G(x, y ; \zeta)$ as a function of $(x, y)$ is defined in $\mathbb{R}^{3} \times \mathbb{R}^{3}$ almost everywhere only. To avoid ambiguity we consider from here on the function $G(x, y ; \zeta)$ as pointwise sum of the series (6) if $x \neq y$.

(2) The formal representation of the function $G$ in the form (6) has been obtained in [25] without proof of convergence of the series (6) in any sense.

\section{Auxiliary results}

At first we introduce the notation $\mathbb{R}_{\perp}^{3}=\left\{x \in \mathbb{R}^{3}: x_{\perp} \neq 0\right\}$. Consider the integrals

$$
\begin{aligned}
& J(x ; \zeta)=\int_{-\infty}^{+\infty} \Gamma\left(\frac{1}{2}-\frac{\zeta-p^{2}}{\omega}\right) \Psi\left(\frac{1}{2}-\frac{\zeta-p^{2}}{\omega}, 1 ; \pi \xi x_{\perp}^{2}\right) \exp \left(i p x_{3}\right) d p \\
& J_{1}(x ; \zeta)=\int_{-\infty}^{+\infty} \int_{0}^{+\infty} \exp \left(i p x_{3}-\pi \xi x_{\perp}^{2} t\right)\left(\frac{t}{1+t}\right)^{\frac{1}{2}-\frac{\zeta-\rho^{2}}{\omega}} \frac{d t d p}{t}
\end{aligned}
$$

where $x \in \mathbb{R}^{2} \backslash\{0\}$ and $\zeta \in \rho(H)$.

Lemma 2. For any $\delta>0$ the integrals (16) and (17) converge absolutely and locally uniformly in the region $\left|x_{\perp}\right| \geq \delta$ and $\operatorname{Re} \zeta \leq \frac{\omega}{2}-\delta$. Therefore, the functions $J$ and $J_{1}$ are jointly continuous in $(x, \zeta)$ and $J(x ; \zeta)=J_{1}(x ; \zeta)$.

Proof. Because of equation (3) it suffices to prove that the integral (17) converges absolutely and uniformly in the mentioned region. It is evident that the assertion follows from the estimate

$$
\int_{-\infty}^{+\infty} \int_{0}^{+\infty} \exp (-\beta t)\left(\frac{t}{1+t}\right)^{\alpha+q^{2}} \frac{d t d q}{t}<+\infty
$$


where $\alpha>0$ and $\beta>0$. Using the well-known formula

$$
\int_{-\infty}^{+\infty} e^{-\lambda x} e^{-k x^{2}} d x=\sqrt{\frac{\pi}{k}} e^{-\frac{\lambda^{2}}{4 k}} \quad(\lambda \in \mathbb{C}, k>0)
$$

we get

$$
\int_{-\infty}^{+\infty}\left(\frac{t}{1+t}\right)^{q^{2}} d q=\sqrt{\pi}\left(\ln \frac{1+t}{t}\right)^{-\frac{1}{2}}
$$

Because

$$
\left(\ln \frac{1+t}{t}\right)^{-\frac{1}{2}}=o(1)(t \rightarrow 0+) \text { and }\left(\ln \frac{1+t}{t}\right)^{-\frac{1}{2}}=O\left(t^{\frac{1}{2}}\right)(t \rightarrow+\infty)
$$

we obtain the estimate (18)

Lemma 3. Let $x \in \mathbb{R}_{\perp}^{3}$ and $\operatorname{Re} \zeta<\frac{\omega}{2}$. Then

$$
J(x ; \zeta)=2 \pi \sqrt{\xi} \int_{0}^{+\infty} \frac{\exp \left\{-\pi \xi\left[\left(x_{\perp}-y_{\perp}\right)^{2}\left(e^{t}-1\right)^{-1}+\left(x_{3}-y_{3}\right)^{2} t^{-1}\right]\right\}}{\exp \left(\left(\frac{1}{2}-\frac{\zeta}{\omega}\right) t\right)\left(1-e^{-t}\right)} \frac{d t}{\sqrt{t}} .
$$

Moreover, the integral in the right-hand side converges locally uniformly in the domain $\left(\mathbb{R}^{3} \backslash\{0\}\right) \times\left\{\zeta \in \mathbb{C}: \operatorname{Re} \zeta<\frac{\omega}{2}\right\}$.

Proof. Lemma 2 makes possible to interchange in (17) the order of integration. Using equation (19), we obtain

$$
J(x ; \zeta)=2 \pi \sqrt{\xi} \int_{0}^{+\infty}\left(\frac{t}{1+t}\right)^{\frac{1}{2}-\frac{\varsigma}{\omega}} \exp \left[-\pi \xi\left(x_{\perp}^{2} t+\frac{x_{3}^{2}}{\ln \frac{1+t}{t}}\right)\right] \frac{d t}{t\left[\ln \left(\frac{1+t}{t}\right)\right]^{\frac{1}{2}}}
$$

The change of variable $\frac{t}{1+t}=\exp (-u)$ yields the right-hand side of (21). Obviously, the integral (21) converges on the upper limit of integration absolutely and uniformly in any set of the form $\mathbb{R}^{3} \times\left\{\zeta \in \mathbb{C}: \operatorname{Re} \zeta \leq \frac{\omega}{2}-\delta\right\}$ where $\delta>0$. If $u>0$ is sufficiently small, then $e^{u}-1 \leq 2 u$, therefore for all $u$ belonging to a sufficiently small interval $(0, \varepsilon)$

$$
\exp \left(-\pi \xi\left(x_{\perp}^{2}\left(e^{u}-1\right)^{-1}+x_{3}^{2} u^{-1}\right)\right) \leq \exp \left(-\frac{\pi \xi}{2} u^{-1} x^{2}\right)
$$

Moreover, for all $(x, \zeta)$ with $|x| \geq \delta$ and $\operatorname{Re} \zeta<\frac{\omega}{2}$ the integral (21) is majorized on the lower limit of integration by the concergent integral

$$
J_{1} \equiv \int_{0}^{\varepsilon} \exp \left(-\frac{\pi \xi}{2} \delta^{2} u^{-1}\right) \frac{d u}{\left(1-e^{-u}\right) \sqrt{u}}
$$

Hence the lemma is proved 
Lemma 4. If $\operatorname{Re} \zeta<\frac{\omega}{2}$, then for every $C>0$

$$
\int_{|x|<C}|J(x ; \zeta)| d x<+\infty .
$$

Proof. We have with some $\alpha>0$ and $\beta>0$

$$
\begin{aligned}
\int_{|x|<C}|J(x ; \zeta)| d x & \leq 2 C \int_{\left|x_{\perp}\right|<C} \int_{-\infty}^{+\infty} \int_{0}^{+\infty} \exp \left(-\beta x_{\perp}^{2} t\right)\left(\frac{t}{1+t}\right)^{\alpha+q^{2}} \frac{d x_{\perp} d t d q}{t} \\
& =2 C \frac{\pi}{\beta} \int_{-\infty}^{+\infty} \int_{0}^{+\infty}\left[1-\exp \left(-\beta C^{2} t\right)\right]\left(\frac{t}{1+t}\right)^{\alpha+q^{2}} \frac{d t d q}{t^{2}}
\end{aligned}
$$

Because of (20) the estimate (23) implies (22)

Lemma 5 below provides a possibility to continue analytically the function $J(x ; \zeta)$ on the domain $\rho(H)$.

Lemima 5. Let $n \in \mathbb{N}, a \in \mathbb{C}$ and $x>0$. If $a-n \notin \mathbb{Z}_{-}$, then for any $n \in \mathbb{N}$

$$
\begin{aligned}
\Gamma(a-n) \Psi(a-n, 1 ; x) \\
=\sum_{k=0}^{n} s_{k}(a) x^{k} \Gamma(a) \Psi(a, 1 ; x)+\sum_{k=0}^{n} t_{k}(a) x^{k} \Gamma(a+1) \Psi(a+1,1 ; x)
\end{aligned}
$$

where $s_{k}$ and $t_{k}$ are rational functions, i.e functions having the form $\frac{P}{Q}$ for polynomials $P$ and $Q$ obeying $\operatorname{deg} P \leq \operatorname{deg} Q$ and $Q$ having only the numbers $1, \ldots, n$ as possible roots. If $n=0$, then we can take $s_{0}=1$ and $t_{0}=0$.

Proof. To prove the lemma we need the formula

$$
\Psi(a-1,1 ; x)=(2 a-1+x) \Psi(a, 1 ; x)-a^{2} \Psi(a+1,1 ; x)
$$

(see [4: Vol.1/Formula 6.6(4)]). It implies

$$
\Gamma(a-1) \Psi(a-1,1 ; x)=\frac{2 a-1+x}{a-1} \Gamma(a) \Psi(a, 1 ; x)-\frac{a}{a-1} \Gamma(a+1) \Psi(a+1,1 ; x) .
$$

By induction we obtain

$$
\begin{aligned}
\Gamma(a-n) \Psi(a-n, 1 ; x)= & \frac{2 a-2 n+1+x}{a-n} \Gamma(a-n+1) \Psi(a-n+1,1 ; x) \\
& -\frac{a-n+1}{a-n} \Gamma(a-n+2) \Psi(a-n+2,1 ; x)
\end{aligned}
$$

if $a-n \notin \mathbb{Z}$. . The lemma is immediate from this equation

Using Lemma 5, we deduce from Lemmas 2 and 4 by standard arguments the following two lemmas.

Lemma 6. The integral (16) converges locally unifirmly in the domain $\mathbb{R}_{\perp}^{3} \times \rho(H)$. Moreover, for every $\varepsilon>0$ and compact subset $K$ of $\rho(H)$ there exist $C>0$ and $m \in \mathbb{N}$ such that $|J(x ; \zeta)| \leq\left(1+x_{\perp}^{2}\right)^{m}$ if $\left|x_{\perp}\right| \geq \varepsilon$ and $\zeta \in K$. In particular, for every $\zeta \in \rho(H)$ the function $J(\cdot ; \zeta)$ is continuous in the domain $\mathbb{R}_{\perp}^{3}$

Lemma 7. Let $C>0$. Then estimate (22) holds for each $\zeta \in \rho(H)$ 


\section{Integral representations and asymptotics of $G(x, y ; \zeta)$}

At the beginning of the section we get another representation of the function $G$.

Theorem 2. Let $\zeta \in \rho(H)$. Then the following assertions are valid:

(i) For every $(x, y) \in \mathbb{R}_{\Delta}^{6}$ obeying $x_{\perp} \neq y_{\perp}$ we have

$$
G(x, y ; \zeta)=\frac{1}{8 \pi^{2}} \Phi(x, y) J(y-x ; \zeta) .
$$

(ii) For every $(x, y) \in \mathbb{R}_{\Delta}^{6}$ we have

$$
\begin{aligned}
G(x, y ; \zeta)= & \frac{\xi^{\frac{1}{2}}}{4 \pi} \Phi(x, y) \\
& \times \int_{0}^{+\infty} \frac{\exp \left\{-\pi \xi\left[\left(x_{\perp}-y_{\perp}\right)^{2}\left(e^{t}-1\right)^{-1}+\left(x_{3}-y_{3}\right)^{2} t^{-1}\right]\right\}}{\exp \left(\left(\frac{1}{2}-\frac{\zeta}{\omega}\right) t\right)\left(1-e^{-t}\right)} \frac{d t}{\sqrt{t}} .
\end{aligned}
$$
obtain

Proof. Applying the Fourier transform with respect to $x_{3}$ to the operator (1), we

$$
H=H^{(2)} \otimes I_{p}+I_{x_{1} x_{2}} \otimes p^{2} .
$$

Needless to say that $p^{2}$ denotes the corresponding multiplication operator in the space $L^{2}\left(\mathbb{R}_{p}\right)$ of the variable $p$. Therefore for all $f \in L^{2}\left(\mathbb{R}^{3}\right)$ and $\zeta \in \rho(H)$ we have

$$
\begin{aligned}
(H-\zeta)^{-1} f(x)= & \frac{1}{2 \pi} \int_{-\infty}^{+\infty} e^{i p x_{3}} d p \\
& \times \int_{\mathbb{R}^{2}} G^{(2)}\left(x_{\perp}, y_{\perp} ; \zeta-p^{2}\right) d y_{\perp} \int_{-\infty}^{+\infty} e^{-i p y_{3}} f\left(y_{\perp}, y_{3}\right) d y_{3} .
\end{aligned}
$$

The integrals with respect to $p$ and $y_{3}$ converge here in $L^{2}$-norm. Denote temporarily $\frac{1}{8 \pi^{2}} \Phi(x, y) J(y-x ; \zeta)$ by $\widetilde{G}(x, y ; \zeta)$. Let $\zeta \in \rho(H)$ be fixed. If a function $f \in L^{2}\left(\mathbb{R}^{3}\right)$ has compact support, then by Lemma 7 the integral (27) converges absolutely and hence

$$
\int_{\mathbb{R}^{3}} G(x, y ; \zeta) f(y) d y=\int_{\mathbb{R}^{3}} \widetilde{G}(x, y ; \zeta) f(y) d y .
$$

Therefore $G(x, y ; \zeta)=\widetilde{G}(x, y ; \zeta)$ for almost all $(x, y) \in \mathbb{R}^{3} \times \mathbb{R}^{3}$. By continuity $G(x, y ; \zeta)$ $=\widetilde{G}(x, y ; \zeta)$ for all $(x, y) \in \mathbb{R}_{\Delta}^{6}$ obeying $x_{\perp} \neq y_{\perp}$. Thus part (i) of the theorem is proved. Part (ii) follows from Lemma 3

Theorem 3. Let $\zeta_{0} \in \rho(H)$. Then for every $\varepsilon>0$ there exist constants $\delta>0$ and $C_{1}, C_{2}>0$ such that

$$
|G(x, y ; \zeta)| \leq C_{1} \exp \left(-C_{2}|x-y|\right)
$$

if $|x-y| \geq \varepsilon$ and $\left|\zeta_{0}-\zeta\right| \leq \delta$.

Proof. Let $\varepsilon>0$ be given. If $|x-y| \geq \varepsilon$, then either $\left|x_{\perp}-y_{\perp}\right| \geq \frac{\varepsilon}{2}$ or $\left|x_{\perp}-y_{\perp}\right|<\frac{\varepsilon}{2}$ and $\left|x_{3}-y_{3}\right| \geq \frac{\varepsilon}{2}$. Therefore the theorem follows from Lemmas 8 and 9 below 
Lemma 8. Let $\zeta_{0} \in \rho(H)$. Then for every $\varepsilon>0$ there exist constants $\delta>0$ and $C_{1}, C_{2}>0$ such that

$$
|G(x, y ; \zeta)| \leq C_{1} \exp \left(-C_{2}\left|x_{\perp}-y_{\perp}\right|\right)
$$

if $\left|\zeta-\zeta_{0}\right| \leq \delta$ and $\left|x_{\perp}-y_{\perp}\right| \geq \varepsilon$.

Proof. Using (25) and (5), we obtain the assertion from Lemma 6

Lemma 9. Let $\zeta_{0} \in \rho(H)$. Then for every $\varepsilon>0$ there exist constants $\delta$ and $C_{1}, C_{2}>0$ such that

$$
|G(x, y ; \zeta)| \leq C_{1} \exp \left(-C_{2}\left|x_{3}-y_{3}\right|\right)
$$

if $\left|\zeta-\zeta_{0}\right| \leq \delta,\left|x_{\perp}-y_{\perp}\right| \leq \varepsilon$ and $\left|x_{3}-y_{3}\right| \geq \varepsilon$.

Proof. Pick $\delta>0$ such that the disk $\left|\zeta-\zeta_{0}\right| \leq \delta$ do not intersect $\sigma(H)$. By Theorem 1 and equation (8) we have

$$
|G(x, y ; \zeta)| \leq C_{0} \sum_{l=0}^{\infty} \frac{\exp \left(-\widetilde{C}\left|x_{3}-y_{3}\right|\right)}{\left|\sqrt{\zeta-\varepsilon_{l}}\right|}
$$

where

$$
\widetilde{C}=\inf \left\{\operatorname{Re} \sqrt{l+\frac{1}{2}-\frac{\zeta}{\omega}}:\left|\zeta-\zeta_{0}\right| \leq \delta \text { and } l \in \mathbb{N}\right\} .
$$

It is clear that for $\delta$ sufficiently small we have that $\tilde{C}>0$. If $\widetilde{C}>C_{2}>0$, then

$$
\begin{aligned}
|G(x, y ; \zeta)| & \leq C_{0} \exp \left(-C_{2}\left|x_{3}-y_{3}\right|\right) \sum_{l=0}^{\infty} \frac{\exp \left(-\left(\widetilde{C}-C_{2}\right)\left|x_{3}-y_{3}\right|\right)}{\left|\sqrt{\zeta-\varepsilon_{l}}\right|} \\
& \leq C_{1} \exp \left(-C_{2}\left|x_{3}-y_{3}\right|\right)
\end{aligned}
$$

and the assertion is proved

We want to give in conclusion an application of the obtained results to the finding of zero-range perturbations of the three-dimensional Landau operator. Namely, we give a simple derivation of the diagonal elements $Q_{\alpha, \alpha}(z)$ of the Krein $Q$-matrix for the operator $H$. Because of analyticity in $z, Q_{\alpha, \alpha}(z)$ can be defined up to addive constant by the relation

$$
\frac{\partial Q_{\alpha, \alpha}(z)}{\partial z}=\frac{\partial G(\alpha, \alpha ; z)}{\partial z}
$$

where $\operatorname{Re} z<\frac{\omega}{2}$ (see [17]). From equation (26) we get

$$
\left.\frac{\partial}{\partial z} G(x, y ; z)\right|_{x=y}=\frac{1}{16 \pi^{2} \sqrt{\xi}} \int_{0}^{+\infty} \frac{\exp \left\{-\left(\frac{1}{2}-\frac{z}{\omega}\right) t\right\}}{1-e^{-t}} \sqrt{t} d t .
$$

Using the equality

$$
\int_{0}^{+\infty} t^{s-1} e^{-v t}\left(1-e^{-t}\right)^{-1} d t=\Gamma(s) \zeta(s, v)
$$


where $\zeta=\zeta(s, v)$ is the so-called generalized Riemann $\zeta$-function (see [4: Vol. $1 /$ Subsection 1.10]) and the relation

$$
\frac{\partial}{\partial s} \zeta(s, v)=-s \zeta(s, v)
$$

we obtain at once

$$
Q(\alpha, \alpha ; z)=\frac{\sqrt{\xi}}{4} \zeta\left(\frac{1}{2} ; \frac{1}{2}-\frac{z}{\omega}\right)+\text { const. }
$$

We remark that this equation has been obtained at a physical level of rigour in [7: Section VII.2/Formula (VII.2.9)].

Acknowledgments. The work is partly supported by grant No 94-2.7-1067 of the Ministry of Higher Education of Russia and RFFR grants No 95-01-00439 and No 9502-04871.

\section{References}

[1] Albeverio, S., Gesztesy, F., Høegh-Krohn, R. and H. Holden: Solvable Models in Quantum Mechanics. Berlin - Heidelberg - New York: Springer-Verlag 1988.

[2] Avishai, Y., Azbel, M. Ya. and S. A. Gredeskul: Electon in a magnetic field interacting with point impurities. Phys. Rev. B. 48 (1994), 17280 - 17295.

[3] Bellissard, J., Elst, A. van and H. Schulz-Baldes: The noncommutative geometry of the quantum Hall effect. J. Math. Phys. 35 (1994), 5373 - 5451.

[4] Beytman, H. and A. Erdélyi: Higher transcendental functions. Vols. 1 and 2. New York: McGraw-Hill 1953.

[5] Cycon, H. L., Fröse, R. G., Kirsch, W. and B. Simon: Schrödinger Operators with Applications to Quantum Mechanics and Global Geometry. Berlin - Heidelberg - New York: Springer-Verlag 1987.

[6] Daumer, F.: Équation de Schrödinger avec champ électrique périodique et champ magnétique constant dans l'approximation du tight-binding. Comm. Part. Diff. Equ. 18 (1993), 1021 - 1041.

[7] Demkov, Yu. N. and V. N. Ostrovskiy: Zero-Range Potentials and their Applications in Atomic Physics. New York: Plenum Press 1988.

[8] Gesztesy, F., Holden, H. and P. Seba: On point interaction in magnetic field system. In: Schrödinger Operators, Standard and Nonstandard (eds.: P. Exner and P.Seba). Singapore: World Sci. 1989, pp. $147-166$.

[9] Geyler, V. A.: The two-dimensional Schrödinger operator with a uniform magnetic field, and its perturbation by periodic zero-range potentials. St. Petersburg Math. J. 3 (1992), $489-532$.

[10] Geyler, V. A. and V. V. Demidov: The spectrum of the three-dimensional Landau operators with periodic point perturbations (in Russian). Teoret. i matem. fizika. 103 (1995), 283 294. Engl transl. in: Theor. Math. Phys. 103 (1995), $561-569$.

[11] Geyler, V. A. and V. A. Margulis: Anderson localization in the nondiscrete Maryland model. Theor. Math. Phys. 70 (1987), 133 - 140. 
[12] Geyler, V. A. and V. A. Margulis: Density of states of two-dimensional electrons in the presence of a magnetic field and a random potentials in exactly solvable models. Sov. Phys. JETP. 68 (1989), $654-660$.

[13] Geyler, V. A., Margulis, V. A. and I. I. Chuchaev: Zero-range potentials and Carleman operators (in Russian). Sib. math. zhurn. 36 (1995), $828-841$.

[14] Geyler, V. A., Margulis, V. A., Chudaev, I. V. and I. I. Chuchaev: Conductivity of an electron gas in a quantizing magnetic field with scattering on point defects. JETP. 80 (1995), $100-104$.

[15] Gountarulis, G.: Green-function of the free electron in a uniform magnetic field. Phys. Lett. A. 36 (1972), $132-134$.

[16] Gredeskul, S. A., Avishai, Y. and M. Ya. Azbel: Extended states on the Landau levels with disorder. Europhys. Lett. 21 (1993), $489-494$.

[17] Helffer, B. and J. Sjöstrand: Semi-classical analysis for Harper's equation. Part III. Mém. Soc. Math. France. 39 (1989), 1 - 124.

[18] Hunziker, W.: Schrödinger operators with electric or magnetic fields. Lect. Notes Phys. 116 (1980), $25-44$.

[19] Klitzing, K. von, Dorda, G. and M. Pepper: New method for high-accuracy determination of the fine-structure constant based on quantized Hall resistance. Phys. Rev. Lett. 45 (1980), $494-497$.

[20] Korotkov, V. B.: Integral operators (in Russian). Novosibirsk: Nauka 1983.

[21] Kreĭn, M. G. and H. Langer: On defect numbers and generalized resolvents of an Hermitian operator in the space $\Pi_{\kappa}$. Funct. Anal. Appl. 5 (1971), $59-71$.

[22] Olver, F. W. J.: Asymptotics and Special Functions. New York: Academic Press 1974.

[23] Pavlov, B. S.: The theory of extensions and explicitly-solvable models. Russ. Mat. Surv. 42 (1987), $127-168$.

[24] Prange, R. E. and S. M. Girvin (eds.): The Quantum Hall Effect. Berlin - Heidelberg New York: Springer-Verlag 1987.

[25] Thouless, D. J.: Topological interpretation of quantum Hall effect. J. Math. Phys. 35 (1994), $5362-5372$.

[26] Treves, F.: Introduction to Pseudodifferential and Fourier Integral Operators. New York: Plenum Press 1982. 